\title{
Evidências de Validade da Versão Brasileira da Escala Amor do Marriage and Relationships Questionnaire (MARQ)
}

\author{
Priscilla Soares de Franca - Universidade Federal do Rio Grande do Norte, Natal, Brasil \\ Jean Carlos Natividade - Pontifícia Universidade Católica do Rio de Janeiro, Rio de Janeiro, Brasil \\ Fívia de Araújo Lopes - Universidade Federal do Rio Grande do Norte, Natal, Brasil
}

\begin{abstract}
Resumo
O amor romântico é um sentimento relacionado à criação e manutenção de laços afetivos. Entre os instrumentos desenvolvidos para aferi-lo, destaca-se o Marriage and Relationships Questionnaire por sua abrangência e replicabilidade em diversas culturas. Ele contém 12 escalas, entre as quais, uma afere o nível de vínculo emocional entre parceiros em relacionamento amoroso: a Escala Amor. Este trabalho teve como objetivo buscar evidências de validade da versão adaptada para o Brasil da Escala Amor. Participaram da pesquisa 176 pessoas em relacionamento estável. Realizou-se uma análise fatorial exploratória e verificou-se adequação da estrutura unifatorial do instrumento, além de satisfatória consistência interna e relações com questões critério, conforme hipotetizadas. A escala foi capaz de detectar maiores níveis de vínculo emocional entre as pessoas que nunca pensaram em se separar de seus parceiros, quando comparadas àquelas que pensaram com maior frequência. Considera-se que o instrumento adaptado se mostrou adequado para utilização no Brasil.

Palavras-chave: relacionamento, validade do teste, vínculo emocional, psicometria, psicologia evolucionista
\end{abstract}

Validity Evidences of Brazilian Version of the Love Scale of Marriage and Relationships Questionnaire (MARQ)

\begin{abstract}
Love is a feeling that includes different emotions and is responsible for the creation and maintenance of attachment bonds. Among the instruments developed to measure love, the Marriage and Relationship Questionnaire is noteworthy by its scope and replicability in different cultures. This questionnaire has 12 scales, of which the Love Scale was developed to assess the level of emotional attachment between partners in a romantic relationship. Our goal was to validate the Love Scale as an alternative measure of emotional attachment for Brazilian population. In this study, 176 individuals in a stable relationship participated. An exploratory factor analysis was conducted and verified one factor with high internal consistency, and relations with criteria questions as hypothesized. The scale was able to detect higher levels of emotional attachments between people who never thought to divorce compared to the ones who thought it more frequently. The adapted instrument showed satisfactory psychometrics properties for use in Brazil.

Keywords: relationship, test validity, emotional bond, psychometrics, evolutionary psychology
\end{abstract}

Evidencias de Validez de la Versión Brasileña de la Escala Amor del Marriage and Relationships Questionnaire (MARQ)

\begin{abstract}
Resumen
El amor romántico es un sentimiento relacionado a la creación y mantenimiento de lazos afectivos. Entre los instrumentos desarrollados para evaluarlo, se destaca el Marriage and Relationships Questionnaire por su amplitud y replicabilidad en diversas culturas. Este instrumento contiene 12 escalas, incluyendo una que chequea el nivel de vínculo emocional entre los individuos en una relación amorosa: la Escala Amor. Este trabajo tuvo como objetivo buscar evidencias de validez de la versión adaptada de la Escala Amor para Brasil. Participaron 176 personas que estaban en una relación estable. A partir del análisis factorial exploratorio, se verificó la adecuación de la estructura unifactorial del instrumento, además de satisfactoria consistencia interna y relaciones con cuestiones criterio, que se tenían como hipótesis. La escala fue capaz de detectar niveles elevados de vínculos afectivos entre las personas que nunca han pensado en separarse de sus parejas, comparadas con las que pensaban con mayor frecuencia. Se considera que el instrumento adaptado se mostró adecuado para su utilización en Brasil.

Palabras clave: relación, validez del test, vínculo emocional, psicometría, psicología evolutiva
\end{abstract}

Os relacionamentos românticos estão presentes na vida de quase todas pessoas. A compreensão deles envolve desde investigações sobre os processos de escolha (Botwin, Buss, \& Shackelford, 1997; Broemer \& Diehl, 2003; Buss, 2006; Buston \& Emlen, 2003; Campbell, Simpson, Kashy, \& Fletcher, 2001; Castro, Hattori, \& Lopes, 2012; Castro \& Lopes, 2011; Hattori, Castro, \&
Lopes, 2013; Noë \& Hammerstein, 1994; Pawlowsky \& Dunbar, 1999; Schmitt, Shackelford, Duntley, \& Tooke, 2001) até estudos sobre a manutenção e satisfação nos relacionamentos (Banfield \& McCabe, 2001; Buckle, Gallup, \& Rodd, 1996; Buss \& Schmitt, 1993; Buss \& Schackelford, 1997; Contreras, Hendrick, \& Hendrick, 1996; Gottman \& Krokoff, 1989; Hendrick, Hendrick, 
\& Adler, 1988; Lucas et al., 2008; Murray, Holmes, Dolderman, \& Griffin, 1999; Shackelford, Goetz, \& Buss, 2005). Dentro desse último enfoque, um dos aspectos investigados diz respeito ao sentimento estabelecido entre os indivíduos que compõe o relacionamento: o amor romântico.

Talvez pelo fato de o amor romântico poder apresentar diferentes significados para pessoas distintas em variados relacionamentos em pontos distintos no tempo, tal como proposto por Hendrick e Hendrick (1989), a relutância em mensurá-lo de forma mais categórica tenha perdurado por muito tempo. Por outro lado, e a despeito de possíveis divergências em termos de significados, muitos estudos apontam para o amor romântico como um sentimento universal (Jankowiak \& Fisher, 1992; Landis \& O'Shea III, 2000; Schmitt, 2006) e que, como tal, tem merecido a atenção de diversos pesquisadores enquanto construto.

De acordo com Gouveia, Carvalho, Santos e Almeida (2013), o amor se revela como "forma particularizada de sentimento, quando uma pessoa deseja e busca, de outra pessoa, receber e dar prazeres ou satisfações que podem ser de diferentes naturezas, como sexuais, de admiração, de compreensão e de proteção" (pp. 33). Numa perspectiva psicobiológica, o amor romântico está ligado a muitos aspectos da reprodução humana, sendo responsável pela criação e manutenção de laços afetivos entre indivíduos, de comportamentos de cuidado, proteção do parceiro, e desejo sexual, que podem variar de acordo com as condições ecológicas, sociais, culturais e psicológicas (Shiramizu \& Lopes, 2013). Diante disso, pode-se compreender o amor romântico como um sentimento responsável pelo estabelecimento de um vínculo emocional entre duas pessoas.

Do ponto de vista da Psicologia, pode-se citar duas teorias que propõem definir os aspectos cognitivos do amor - Teoria das Cores (Lee, 1973) e multidimensionais do amor - Teoria Triangular (Sternberg, 1986). Jonh Lee (1973) classificou o amor em seis estilos, os três primeiros baseados na ideia de cores primárias e os demais a partir da combinação dos primários: Eros (amor passional), Ludus (amor como jogo), Storge (amor baseado na amizade), Pragma (amor lógico), Mania (amor obsessivo), Ágape (amor altruísta). Robert Sternberg (1986) por sua vez, propôs um modelo com três componentes, sendo cada um, parte de um triângulo, criando sete possibilidades de amor a partir da combinação dos três vértices: Intimidade, Paixão e Comprometimento. Assim, de acordo com Sternberg
(1986) tem-se o amor pleno, amor romântico, amor companheiro, amor fatual, amor passageiro, amor do tipo gostar e amor vazio.

\section{Mensuração do Amor}

Há diversas escalas que medem vínculo emocional/amor em humanos (Triangular Love Scale - Sternberg, 1986; Love Attitude Scale - Hendrick \& Hendrick, 1986; Love Scale [Marriage and Relationship Questionnaire] - Russel \& Wells, 1993; Tetrangular Model - Yela, 2006). Cassepp-Borges e Andrade (2013), numa revisão sobre instrumentos que se propõem a medir o amor romântico em língua portuguesa, afirmam que "as dificuldades inerentes à sua mensuração são praticamente as mesmas dificuldades inerentes à medida de outros construtos psicológicos" (pp. 621). Nesse sentido, em português, as escalas mais utilizadas são a escala Triangular do Amor (Andrade, Garcia, \& Cassep-Borges, 2013; Cassep-Borges \& Teodoro, 2007) focada nos aspectos multidimensionais do amor, e a Escala de Estilos de Amor (Andrade \& Garcia, 2009) designada para medir os aspectos cognitivos do amor.

Uma opção ainda desconhecida no Brasil para medir esse vínculo emocional é o Marriage and Relationship Questionnaire (MARQ) (Russel \& Wells, 1986). Essa escala é focada em medir o amor romântico em casais em relacionamento estável e possui a praticidade de medir vários aspectos do relacionamento em um único questionário. O MARQ foi originalmente desenvolvido em inglês para fornecer uma visão mais ampla dos sentimentos e emoções na perspectiva dos próprios participantes e de seus relacionamentos com seus cônjuges (Russell \& Wells, 2000). Sua versão inicial incluía 235 questões de verdadeiro/falso e itens de múltipla escolha, cujas respostas estão configuradas em escalas Likert de cinco pontos (Weisfeld, 1997). Sua versão mais atual possui por volta de 190 questões, das quais ao menos 180 são questões fixas. O número de questões pode variar de acordo com a inclusão de questões que englobem a cultura trabalhada (Weisfeld, 1997), e pode derivar mais de 12 escalas formais ou informais (Lucas et al., 2008). Apesar de ser uma escala versátil em medir vários aspectos do relacionamento, um ponto negativo do MARQ é o grande número de perguntas, as quais podem se tornar cansativas, especialmente no âmbito cientifico brasileiro no qual não é permitido motivar os participantes com recompensas monetárias.

O MARQ tem sido utilizado em várias pesquisas envolvendo diferentes culturas para analisar aspectos dos relacionamentos afetivos, entre os quais se podem 
destacar: diferenças e similaridades sexuais entre os cônjuges (Weisfeld et al., 2011), o impacto de filhos na satisfação do relacionamento (Wendorf, Lucas, Imamoglu, Weisfeld, \& Weisfeld, 2011), o efeito cultural e adaptativo da satisfação no relacionamento (Lucas et al., 2008), para predizer violência marital (Russel \& Wells, 2000) e felicidade (Russel \& Wells, 1994), bem como para comparar nível de conflito marital entre culturas (Dillon et al., 2015).

Dado o tamanho do instrumento completo, pesquisadores tendem a trabalhar com um conjunto específico de questões ou escala(s) (por exemplo, Dillon, 2012, Lucas et al., 2008, Weisfeld et al., 2011, Wendorf et al., 2011). Dentre as escalas que recebem atenção dos pesquisadores nos mais variados delineamentos investigativos, pode-se destacar a Escala Amor, desenvolvida para aferir o nível de vínculo emocional com o parceiro de relacionamento amoroso, sendo incluídas as questões de acordo com a proposta original de Russel e Wells (1986). Recentemente, Wendorf et al. (2011) apresentaram evidências de validade dessa escala em três culturas distintas (americanos, britânicos e turcos).

No Brasil, pode-se verificar um crescente interesse pelo estudo do amor romântico (por exemplo, Andrade \& Garcia, 2009, Cassep-Borges \& Teodoro, 2007; Shiramizu \& Lopes, 2013; Shiramizu, Natividade, \& Lopes, 2013). Versões brasileiras de instrumentos para aferir o amor romântico que têm sido utilizados em outras culturas, além de fomentar o desenvolvimento de pesquisas na área no país, podem contribuir para realização de estudos transculturais. Diante disso, a presente pesquisa teve como objetivo apresentar evidências de validade e precisão da Escala Amor do MARQ.

\section{Método}

\section{Participantes}

Participaram 176 adultos, 50\% mulheres, todos se declararam heterossexuais e afirmaram estar em um relacionamento amoroso estável. Trata-se de uma amostra de conveniência em que se buscou um número suficiente de participantes para serem realizadas análises fatoriais posteriormente, considerando que a escala continha nove itens e a recomendação de 10 participantes por itens (c.f. Hair, Anderson, Tatham, \& Black, 2005; Pasquali, 2005). Os participantes afirmaram estar vivendo com seus parceiros durante o período da coleta de dados, com o tempo de convivência variando de 0,25 (três meses) a 51 anos e nove meses $(M=17,0$ anos; $D P$
$=10,4$ anos). Em média, a idade dos homens $(M=44,6$ anos; $D P=11,0$ anos) foi superior a das mulheres $(M$ $=41,1$ anos $D P=9,85$ anos), $t(174)=2,22 ; p=0,028$; $d=0,33$. A escolaridade variou de ensino fundamental incompleto (1,7\% dos participantes) a ensino superior completo $(73,8 \%$ dos participantes).

\section{Instrumento}

A Escala Amor do MARQ (Anexo 1) possui nove itens que são apresentados em forma de pergunta e seguidos por cinco opções ordinais de respostas, tal que 1 significa nem um ponco e 5 corresponde a muito. São exemplos de itens: "você gosta da companhia de sua(seu) parceira(o)?", "Você é feliz com seu relacionamento?".

Além dos itens da Escala Amor, utilizaram-se três questões como critério para evidências de validade discriminante e convergente. As questões foram: "Você já pensou em se separar de sua(seu) parceira(o)?", com cinco opções ordinais de resposta variável entre 1 -pretendo farế-lo a 5 - nunca; "Você se sente sexualmente satisfeito em seu relacionamento?”, com cinco opções ordinais de resposta variável entre 1 - um pouco a 5 - muito; "você se sente próximo(a) de sua(seu) parceira(o)?”, com cinco opções ordinais de resposta variável entre 1 - nem um pouco próximo(a) a 5 - bastante.

\section{Procedimentos}

A escala original em inglês foi traduzida por duas pessoas, brasileiros natos com conhecimento avançado na língua inglesa. As duas traduções foram, então, comparadas pelos pesquisadores e ajustadas quanto à terminologia, sendo realizada uma tradução reversa para atestar que não houve mudanças em relação ao original por um terceiro tradutor. Apresentou-se a versão a 10 estudantes de graduação (Psicologia e Ciências Biológicas) e pós-graduação (Psicobiologia) e solicitou-se que avaliassem a compreensão das questões e, quando necessário, sugerissem reformulações. Foi realizada uma adequação de termos ao cotidiano e realidade local. Depois de realizados os ajustes, concluiu-se a versão do instrumento para prova empírica.

As coletas de dados foram realizadas utilizando o método snowball de recrutamento dos participantes, e durante 15 meses foram distribuídos 400 questionários, dos quais 176 foram devolvidos. Durante a coleta, explicou-se que os cônjuges deveriam ficar separados ou que não conversassem enquanto respondiam o questionário, para que não houvesse influência do cônjuge nas respostas, e que sob nenhuma circunstância 
eles deveriam se consultar para fornecer uma resposta. $\mathrm{O}$ atendimento a tal solicitação foi verificado no recolhimento do questionário nas situações em que não foi possível a permanência do pesquisador durante a coleta. Foi recomendado que o questionário fosse respondido por completo, e que o participante marcasse a alternativa que melhor representasse sua opinião ou que fosse próxima dela. Quando devolvidos ao pesquisador, os questionários foram lacrados em um envelope para manter a anonimidade do casal e, ao mesmo tempo, manter os instrumentos do casal juntos para análises posteriores. Todos os procedimentos éticos foram seguidos em conformidade com a resolução 466/12 do Conselho Nacional de Saúde, sendo o projeto aprovado pelo Comitê de Ética em Pesquisa com Seres Humanos (CAAE nº: 36086112.4.0000.5537).

\section{Resultados}

A fim de testar se os itens correspondentes à Escala Amor da versão traduzida do MARQ poderiam representar um construto unidimensional, referente ao vínculo emocional estabelecido com o parceiro, realizou-se uma análise fatorial exploratória - método Principal Axis. Considerando-se a ausência na literatura de evidências sobre a estrutura do instrumento, julgou-se adequado realizar uma análise fatorial exploratória. Observou-se adequação dos dados à análise proposta: $K M O=0,85$ e teste de esfericidade de Bartlett: $\chi^{2}(36$, $N=176)=479,4 ; p<0,001$. A extração de um único fator, tal como evidenciou scree plot (Figura 1), explicou $38,5 \%$ da variância dos dados. Além do critério de Cattell (1966) para extração de fatores, utilizou-se o critério de Horn (1965). Uma análise paralela de eigenvalues aleatórios demonstrou que o único eigenvalue observado que obteve valor maior que o simulado foi o do fator 1 : eigenvalue observado de 4,04, eigenvalue simulado de 1,46; para o segundo fator o eigenvalue observado foi de 1,01 e o eigenvalue simulado foi de 1,30 (número de itens $=9$, número de casos $=176$, número de amostras bootstrap $=1000$ ). A Tabela 1 mostra as cargas fatoriais e comunalidades dos itens. A consistência interna dos itens foi superior a 0,80 , o que pode ser considerado satisfatório (Cronbach, 1951; Nunnally, 1967).

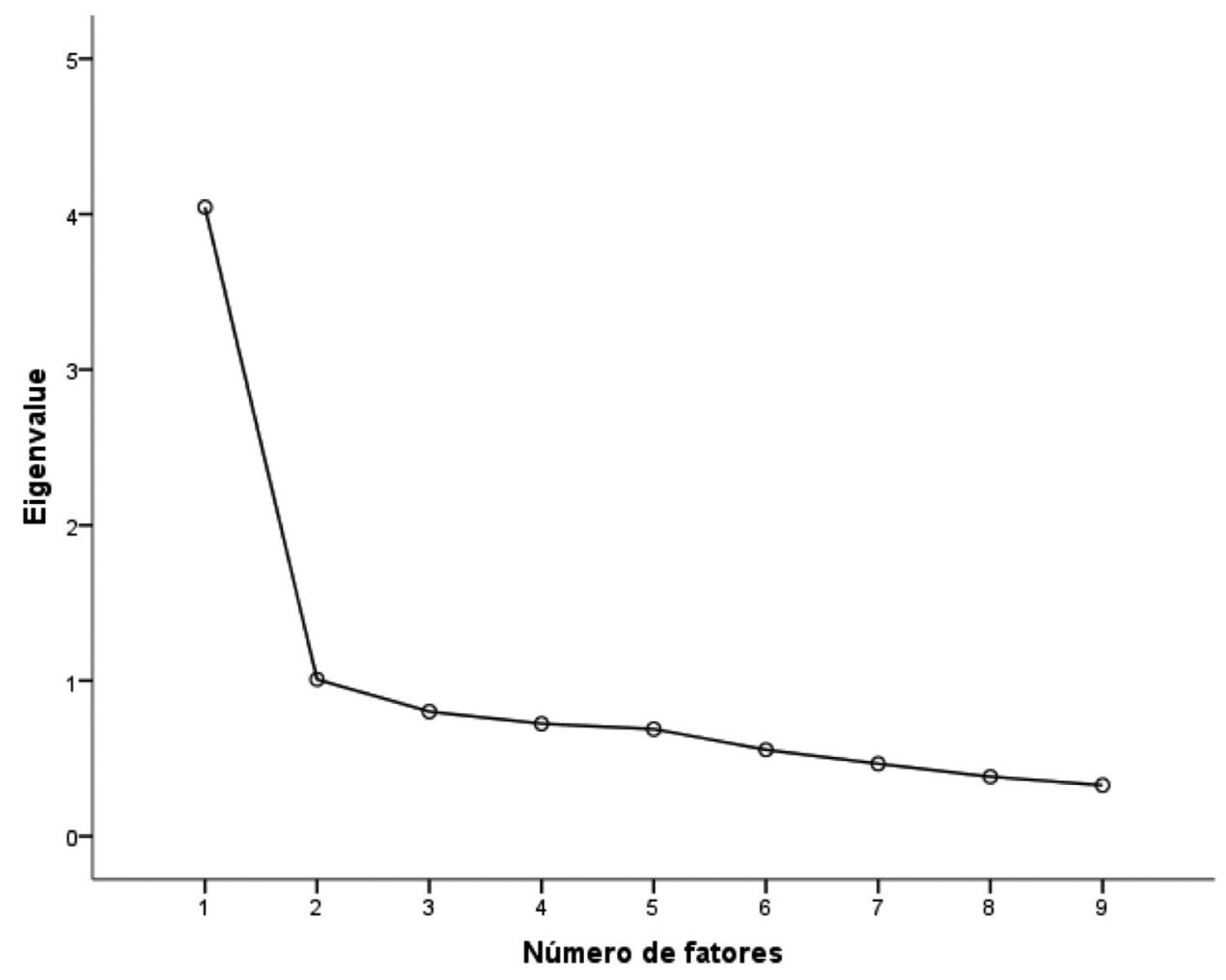

Figura 1. Scree Plot dos itens da versão brasileira da escala Amor do MARQ. 
Calcularam-se média e desvio-padrão para a Escala Amor, que podem ser vistos na Tabela 1. Então, testaram-se correlações entre o vínculo emocional (Escala Amor), idade dos participantes, tempo de relacionamento e as respostas às questões critério "Você se sente sexualmente satisfeito em seu relacionamento?" e "Você se sente próximo(a) de sua(seu) parceira(o)?”. Os resultados da correlação são apresentados na Tabela 2. Destacam-se as correlações positivas e moderadas entre as questões critério e o vínculo emocional, reforçando as evidências de validade do instrumento.

Por fim, a partir da resposta à questão "Você já pensou em se separar de sua(seu) parceira(o)?", formaram-se dois grupos de participantes. Um grupo foi composto por participantes que afirmaram nunca terem pensado em se separar, $n=90$, no outro grupo incluíram-se os participantes que declararam pensar algumas vezes ou frequentemente em separar-se, $n=$ 41. Testou-se o poder do instrumento discriminar esses dois grupos, considerando-se a hipótese de que aqueles que nunca pensaram em se separar apresentariam maiores níveis de vínculo emocional do que aqueles que pensaram algumas vezes e frequentemente. Incluíram-se como covariáveis na ANCOVA a idade dos participantes e o tempo de relacionamento. Além disso, testou-se a interação com a variável sexo. Constatou-se não haver diferenças sexuais no vínculo emocional, $F(1,131)=0,001 ; p=0,98$, tampouco houve interação entre sexo e os grupos formados a partir da questão critério, $F(1,131)=0,035 ; p=0,85$. Contudo, em acordo com a hipótese inicial, houve diferenças significativas nos níveis de vínculo emocional entre aqueles que nunca pensaram em se separar $(M=4,49 ; D P=0,37) \mathrm{e}$ os que pensaram algumas vezes e frequentemente $(M=$ 3,92; $D P=0,50), F(1,131)=53,6 ; p<0,001 ; d=1,30$.

\section{Discussão}

A mensuração do vínculo afetivo presente em uma relação romântica ainda se mostra como um desafio, com uma clara limitação quanto aos instrumentos que se propõe a tal tarefa. O presente estudo teve por objetivo buscar evidências de validade da versão adaptada para o Brasil de uma escala do Marriage and Relationship Questionnaire (MARQ) (Russel \& Wells, 1986). A escala, denominada Escala Amor, propõe-se a avaliar níveis de vínculo emocional entre casais em relacionamento amoroso. Tal como a proposta teórica original do instrumento, encontrou-se um único fator explicando a variação dos itens. Salienta-se que esse resultado pode indicar a primeira evidência na literatura

Tabela 1

Cargas Fatoriais e Comunalidades dos Itens da Escala Amor do MARQ a partir de uma Análise Fatorial com Método Principal Axis

\begin{tabular}{lcc}
\hline Itens & Fator 1 & $h^{2}$ \\
\hline 47. Você é feliz com seu relacionamento? & 0,79 & 0,63 \\
144. Quanto você ama sua(seu) parceira(o)? & 0,70 & 0,48 \\
137. Você se orgulha de sua(seu) parceira(o)? & 0,68 & 0,46 \\
16. Você gosta da companhia de sua (seu) parceira(o)? & 0,64 & 0,41 \\
59. Você gosta de fazer coisas juntos? & 0,60 & 0,36 \\
54. Você acha sua(seu) parceira(o) atraente? & 0,58 & 0,34 \\
133. Você respeita sua(seu) parceira(o)? & 0,57 & 0,32 \\
138. Seu relacionamento tem um lado romântico? & 0,55 & 0,30 \\
105.Você gosta de ficar abraçado(a) com sua(seu) parceira(o)? & 0,17 \\
Eigenvalue & 4,41 & \\
\% Variância explicada & 4,04 & \\
Média & 38,5 & \\
Desvio-padrão & 4,33 & \\
Alfa de Cronbach & 0,46 & \\
\hline
\end{tabular}

Nota. A numeração dos itens segue a ordem da versão completa do Marriage and Relationship Questionnaire (MARQ) (Russel \& Wells, 1986). 
Tabela 2

Correlaçoes de Spearman entre as Variáveis do Estudo

\begin{tabular}{lcccc}
\hline & 1 & 2 & 3 & 4 \\
\hline 1. Vínculo emocional - Escala Amor & -- & & & \\
2. Idade dos participantes & $-0,26^{*}$ & & & \\
3. Tempo de relacionamento & $-0,22^{*}$ & $0,84^{*}$ & & $-0,005$ \\
4. Se sente sexualmente satisfeito? & $0,55^{*}$ & $-0,01$ & $-0,27^{*}$ & $0,43^{*}$ \\
5. Você se sente próximo do parceiro? & $0,63^{*}$ & $-0,26^{*}$ & - \\
\hline
\end{tabular}

Nota. $* p<0,01$; variável 4, $n=175$; variável $5, n=173$; todas as demais variáveis, $n=176$.

sobre a estrutura do instrumento. Considerando-se a definição do construto e a teoria subjacente, a emergência de um único fator sugere adequação estrutural da versão brasileira da Escala Amor do MARQ. Ainda, a consistência interna dos itens mostrou-se satisfatória, denotando precisão ao instrumento.

Adicionalmente, em busca de evidências de validade, testaram-se relações dos níveis de vínculo emocional com as demais variáveis do estudo. Verificaram-se correlações negativas entre vínculo emocional e idade e tempo de relacionamento. Esse resultado vai ao encontro da proposta de Helen Fisher (1992) que sugere que as pessoas tenderiam a se apaixonar por pessoas diferentes após cada ciclo reprodutivo (procriação e cuidado parental inicial), o que pode explicar o desgaste do relacionamento com o tempo. Ainda, de acordo com o trabalho de Wendorf et al. (2011), o vínculo emocional entre o casal diminui intensamente depois de dois ou três anos de relacionamento.

Ainda, observou-se correlação positiva entre níveis de amor romântico e satisfação sexual e percepção de proximidade com o parceiro. Esse resultado está de acordo com as definições de amor de Fisher et al. (2002). De acordo com ela, e conforme descrito anteriormente, o amor romântico é composto por três componentes: atração, luxúria e comprometimento. A luxúria é responsável pelo desejo sexual e motiva os indivíduos a procurarem união sexual. Ela pode ter evoluído como meio de favorecer a satisfação sexual, uma vez que a ovulação em humanos é críptica (Marlowe \& Berbasque, 2012), as mulheres não possuem nenhum sinal comportamental ou fisiológico para indicar a ovulação. Já o comprometimento é responsável pela união emocional, conforto, segurança e manutenção de conexões afiliativas, sendo essencial para a grande maioria dos relacionamentos humanos. Portanto, não é surpresa que a satisfação sexual e a percepção de proximidade sejam positivamente relacionadas com os níveis de amor romântico.

Considerando a possibilidade de separação como mais uma questão critério para testar a validade do instrumento, partiu-se da hipótese de que as pessoas que não pensam em se separar de seus parceiros possuiriam maiores níveis de vínculo emocional e aquelas que pensam em se separar com mais frequência apresentariam menores níveis de vínculo emocional. Tal como esperado, verificou-se que o vínculo emocional tinha níveis mais elevados entre aqueles que não pensaram em separar-se de seus parceiros. Na sociedade ocidental, o amor romântico é tido como um sentimento que possui um conjunto de emoções, as quais são capazes de romper fronteiras e paradigmas e, atualmente, cada vez mais casais são formados e mantidos a partir desse sentimento, e não em favor de opiniões de parentes ou para formar associações econômicas ao juntar duas famílias (Lieberman \& Hatfield, 2006). Hoje, a sociedade permite que duas pessoas que se amam possam viver juntas para formar uma família. Como consequência, há a possibilidade de que divórcios e separações sejam realizados quando o amor romântico entre o casal diminui, evitando que os problemas que surgem com o relacionamento se tornem um fardo para um ou ambos os cônjuges. Dessa forma, o resultado obtido utilizando a perspectiva de separação pode refletir, até certo ponto, que os pensamentos de separação sejam um reflexo da a diminuição do vínculo emocional. No entanto, não se pode perder de vista que o fato da amostra ser composta por casais, em decorrência da interdependência que pode existir nesse contexto específico de coleta, pode ter contaminado positivamente os dados encontrados, o que pode se configurar como uma clara limitação ao presente estudo.

Por fim, destaca-se a carência de instrumentos psicométricos com adequadas evidências de validade para 
o Brasil para utilização em pesquisas que abordem comportamentos reprodutivos. Os resultados apresentados vão de encontro a essa carência mostrando evidências de validade para a Escala Amor do MARQ - Brasil, indicando que a escala pode ser utilizada como uma medida de vínculo emocional em futuras pesquisas brasileiras. Considera-se importante salientar as limitações amostrais deste estudo enquanto representativa da população brasileira, como a quantidade de participantes, idade e escolaridade deles. Incentiva-se a realização de novas pesquisas com a Escala Amor do MARQ Brasil, principalmente, com o intuito de buscarem-se novas evidências de validade em outros contextos brasileiros. Espera-se, ainda, que o instrumento possa abrir portas para futuras pesquisas.

\section{Referências}

Andrade, A. L., \& Garcia, A. (2009). Atitudes e crenças sobre o amor: Versão brasileira da escala de estilos do amor. Interpersona: An International Journal on Personal Relationships, 3, 89-102. doi: 10.1590/ S0102-37722014000100008

Andrade, A. L., Garcia, A., \& Cassep-Borges, V. (2013). Evidências de validade da escala triangular do amor de Sternberg. Psico-USF, 18(3), 501-510. Recuperado de http://www.scielo.br/pdf/pusf/v18n3/ a16v18n3.pdf

Banfield, S., \& McCabe, M. P. (2001). Extra relationship involvement among women: Are they different from men? Archives of Sexual Behavior, 30(2), 119142. doi: 10.1023/A:1002773100507

Botwin, M. D., Buss, D. M., \& Shackelford, T. K. (1997). Personality and mate preferences: Five factors in mate selection and marital satisfaction. Journal of Personality, 65(1), 107-136. doi: 10.1111/j.14676494.1997.tb00531.x

Broemer, P., \& Diehl, M. (2003). What you think is what you get: Comparative evaluations of close relationships. Personality and Social Psychology Bulletin, 29(12), 1560-1569. doi: 10.1177/0146167203256918

Buckle, L., Gallup Jr., \& G. G., Rodd, Z. A. (1996). Marriage as a reproductive contract: Patterns of marriage, divorce, and remarriage. Ethology and Sociobiology, 17, 363-377. Recuperado de http://www.sciencedirect.com/science/ journal/01623095
Buss, D. M. (2006). Strategies of human mating. Psychological Topics, 15(2), 239-260. Recuperado de http://hrcak.srce.hr/index. php?show=clanak\&id_clanak_jezik $=14203$

Buss, D. M., \& Schmitt, D. P. (1993). Sexual strategies theory: An evolutionary perspective on human mating. Psychological Review, 100(2), 204-232. doi: 10.1037/0033-295X.100.2.204

Buss, D. M., \& Shackelford, T. K. (1997). From vigilance to violence: Mate retention tactics in married couples. Journal of Personality and Social Psychology, 22(2), 346-361. doi: 10.1037/0022-3514.72.2.346

Buston, P. M., \& Emlen, S. T. (2003). Cognitive process underlying human mate choice: The relationship between self-perception and mate preference in Western society. Proceeding of the National Academy of Sciences, 100(15), 8805-8810. doi: 10.1073/ pnas. 1533220100

Campbell, L., Simpson, J. A, Kashy, D. A., \& Fletcher, G. J. O. (2001). Ideal standards, the self, and flexibility of ideals in close relationships. Personality and Social Psychology Bulletin, 27(4), 447-462. doi: 10.1177/0146167201274006

Cassep-Borges, V., \& Teodoro, M. L. M. (2007). Propriedades psicométricas da versão brasileira da escala triangular do amor de Sternberg. Psicologia: Reflexão e Critica, 20(3), 513-522. Recuperado de http://www.scielo.br/pdf/prc/v20n3/a20v20n3. pdf

Cassepp-Borges, V., \& Andrade, A. L. (2013). Uma breve história das tentativas para medir atributos dos relacionamentos amorosos em língua portuguesa. Estudos de Psicologia (Natal), 18(4), 621-628. Recuperado de http://www.scielo.br/pdf/epsic/v18n4/ a11v18n4.pdf

Castro, F. N., Hattori, W. T., \& Lopes, F. A. (2012). Relationship maintenance or preference satisfaction? Male and female strategies in romantic partners' choice. Journal of Social, Evolutionary \& Cultural Psychology, 6, 217-226. doi:10.1037/h0099213

Castro, F. N., \&Lopes, F. A. (2011). Romantic preferences in Brazilian undergraduate students: From the short term to the long term. The Journal of Sex Research, 48, 479-485. doi: 10.1080/00224499.2010.506680

Cattell, R. B. (1966). The screen test for the number of factors. Multivariate Behavioral Research, 1, 245-276. 
Contreras, R., Hendrick, S. S., \& Hendrick, C. (1996). Perspectives on marital love and satisfaction in Mexican American and Anglo-American couples. Journal of Counseling \& Development, 74, 408-415. doi: 10.1002/j.1556-6676.1996.tb01887.x

Cronbach, L. (1951). Coefficient alpha and the internal structure of tests. Psychometrika, 16(3), 297-334.

Dillon, L. M. (2012). Sex differences in happiness and marital satisfaction: The contribution of children, attractiveness, and financial status. Dissertation for Doctor Degree at Wayne State University. Detroit, Michigan.

Dillon, L. M., Nowak, N., Weisfeld, G. E., Weisfeld, C. C., Shattuck, K. S., Imamoğlu, O. E. Butovskaya, M., \& Shen, J. (2015). Sources of marital conflict in five cultures. Evolutionary Psychology, 13(1), 1-15. doi: 10.1177/147470491501300101

Fisher, H. (1992). Anatomy of love: The natural history of Monogamy, Adultery and Divorce. New York: Norton.

Fisher, H. E., Aron, A., Mashek, D., Li, H., \& Brown, L. L. (2002). Defining the brain systems of lust, romantic attraction, and attachment. Archives of Sexual Behavior, 31(5), 413-419. Recuperado de http://link.springer.com/ article/10.1023/A:1019888024255

Gottman, J. M., \& Krokoff, L. J. (1989). Marital interaction and satisfaction: A longitudinal view. Journal of Consulting and Clinical Psychology, 57(1), 47-52. doi: 10.1037/0022-006X.57.1.47

Gouveia, V. V., Carvalho, E. A. B., Santos, F. A., \& Almeida, M. R. (2013). Escala tetrangular do amor: Testando sua estrutura e invariância fatorial. Psicologia: Ciência e Profissão, 33(1), 32-45. doi: 10.1590/ S1414-98932013000100004

Hair, J. F. J., Anderson, R. E., Tatham, R. L., \& Black, W. C. (2005). Análise multivariada de dados. Porto Alegre: Bookman.

Hattori, W. T., Castro, F. N., \& Lopes, F. A. (2013). Mate choice in adolescence: Idealizing romantic partners. Psico, 44, 226-234. Recuperado de http:// revistaseletronicas.pucrs.br/ojs/index.php/revistapsico/ article/viewArticle/11466

Hendrick, C., \&Hendrick, S. S. (1986). A theory and method of love. Journal of Personality and Social Psychology, 50, 2, 392-402. doi: 10.1037/0022-3514.50.2.392
Hendrick, C., \& Hendrick, S. S. (1989). Research on love: Does it measure up? Journal of Personality and Social Psychology, 56(5), 784-794. doi: 10.1037/0022-3514.56.5.784

Hendrick, S. S., Hendrick, C., \& Adler, N. L. (1988). Romantic relationships: Love, satisfaction, and staying together. Journal of Personality and Social Psychology, 54(6), 980-988. doi: 10.1037/0022-3514.54.6.980

Horn, J. L. (1965). A rationale and technique for estimating the number of factors in factor analysis. Psychometrika, 30(1), 179-185.

Jankowiak, W. R., \& Fisher, E. F. (1992). A cross-cultural perspective on romantic love. Ethnology, 31(2), 149-157. Recuperado de http://www.jstor.org/ stable/3773618

Landis, D., \& O'Shea III, W. A. (2000). Cross-cultural aspects of passionate love: An individual differences analysis. Journal of Cross-Cultural Psychology, 31(6), 752-777. doi: 10.1177/0022022100031006005

Lee, J. A. (1973). The colors of love. An exploration of the ways of loving. Don Mill, Ont.: New Press.

Lieberman, D., \& Hatfield, E. (2006). Passionate Love: Cross-cultural and evolutionary perspectives. Em R. J. Sternberg \& K. Weiss (Eds.), The New Psychology of Love (pp. 274-297). New Haven, Conn.: Yale University Press.

Lucas, T., Parkhill, M. R., Wendorf, C. A., Imamoglu, E. O., Weisfeld, C. C., \& Weisfeld, G. E. (2008). Cultural and evolutionary components of marital satisfaction: A multidimensional assessment of measurement invariance. Journal of Cross-Cultural Psychology, 39(1), 109-123. doi: 10.1177/0022022107311969

Marlowe, F. W., Berbesque, J. C. (2012). The human operational sex ratio: Effecs of marriage, concealed ovulation, and menopause on mate competition. Journal of Human Evolution, 63(6), 834-842. doi: 10.1016/j.jhevol.2012.09.004

Murray, S. L., Holmes, J. G., Dolderman, D., \& Griffin, D. W. (1999). What the motivated mind sees: Comparing friends perspectives to married partners views of each other. Journal of Experimental Social Psychology, 36, 600-620. doi: 10.1006/jesp.1999.1417

Noë, R., \& Hammerstein, P. (1994). Biological markets: Supply and demand determines the effect 
of partner choice in cooperation, mutualism and mating. Behavioral Ecology and Sociobiology, 35, 1-11. Recuperado de http://link.springer.com/ article/10.1007/BF00167053

Nunnally, J. C. (1967). Psychometric theory. New York, NY: McGraw Hill.

Pasquali, L. (2005). Análise fatorial para pesquisadores. Brasília: LabPAM.

Pawlowsky, B., \& Dunbar, R. I. M. (1999). Impact of market value on human mate choice decisions. Royal Society London, 266, 281-285. doi: 10.1098/ rspb.1999.0634

Russel, R. J. H., \& Wells, P. A. (1993). Marriage and relationships questionnaire: $M A R Q$ bandbook. Jent, Uk: Hodder and Stoughton.

Russell, R. J. H., \& Wells, P. A. (1994). Predictors of happiness in married couples. Personal Individual Differences, 17(3), 313-321. doi:10.1016/0191-8869(94)90279-8

Russell, R. J. H., \& Wells, P. A. (1986). Marriage questionnaire. Unpublished booklet.

Russell, R. J. H., \& Wells, P. A. (2000). Predicting marital violence from the marriage and Relationship Questionnaire: Using LISREL to solve an incomplete data problem. Personality and Individual Differences, 29, 429-440. doi: 10.1016/S0191-8869(99)00203-2

Schmitt, D. P. (2006). Evolutionary and cross-cultural perspectives on love: The influence of gender, personality, and local ecology on emotional investment in romantic relationships. Em R. J. Sternberg \& K. Weiss (Eds.), The New Psychology of Love (pp. 249-273). New Haven, Conn.: Yale University Press.

Schmitt, D. P., Shackelford, T. K., Duntley, J., \& Tooke, W., B. D. M. (2001). The desire for sexual variety as a key to understanding basic human mating strategies. Personal Relationships, 8, 425-455. doi: 10.1111/j.1475-6811.2001.tb00049.x

Shackelford, T. K., Goetz, A. T., \& Buss, D. M. (2005). Mate retention in marriage: Further evidence of the reliability of the mate retention inventory. Personality and Individual Differences, 39, 415-425. doi: 10.1016/j.paid.2005.01.018

Shiramizu, V. K. M., \& Lopes, F. A. (2013). A perspectiva evolucionista sobre relações românticas. Psicologia USP, 24(1), 55-76. Recuperado de http:// www.scielo.br/pdf/pusp/v24n1/v24n1a04.pdf

Shiramizu, V. K. M., Natividade, J. C., \& Lopes, F. A. (2013). Evidências de Validade do Experience in Close Relationships (ECR) Inventory para o Brasil. Estudos de Psicologia (Natal), 18(3), 457-465. doi: 10.1590/S1413-294X2013000300006

Sternberg, R. J. (1986). A triangular Theory of Love. Psychological Review, 93(2), 119-135. doi: 10.1037/0033-295X.93.2.119

Weisfeld, C. C. (1997). Marriage in cross-culture perspective. Em N. L. Segal, G. E. Weisfeld \& C. C. Weisfeld (Eds.), Uniting Psychology and Biology: Integrative Perspectives on Human Development (pp. 355-367). Washington: American Psychology Association.

Weisfeld, C. C., Dillon, L. M., Nowak, N. T., Mins, K. R., Weisfeld, G. E., ... Shen, J. (2011). Sex differences and similarities in married couples: Patterns across and within cultures. Archives in Sexual Behavior, 40, 1165-1172. doi: 10.1007/s10508-011-9790-9

Wendorf, C. A., Lucas, T., Imamoglu, E. O., Weisfeld, C. C., \& Weisfeld, G. E. (2011). Marital satisfaction across three cultures: Does the number of children have an impact after accounting for other marital demographics? Journal of Cross-Cultural Psychology, 42(3), 340-354. doi: 10.1177/0022022110362637

Yela, C. (2006). The evaluation of love: Simplified version of the scales for Yela's tetrangular model based on Stenberg's model. European Journal of Psychological Assessment, 22, 21-27. doi: 10.1027/1015-5759.22.1.21

Recebido em: 08/04/2015 Reformulado em: 19/08/2015 Aprovado em: 24/11/2015 
Sobre os autores:

Priscilla Soares de França é graduada em Ciências Biológicas pela UFRN, mestre em Psicobiologia pela mesma instituição. Seus interesses de pesquisa são: estratégias sexuais, seleção de parceiros, preferências masculinas, preferências femininas, satisfação marital e infertilidade.

E-mail: franca.priscilla18@gmail.com

Jean Carlos Natividade é graduado em Psicologia pela UFSC, com mestrado pela mesma instituição e Doutorado pela UFRGS e professor do Departamento de Psicologia da PUC-RJ. Atualmente, os interesses de pesquisa são nas áreas: autoconceito, personalidade e memória de pessoas, estratégias reprodutivas e relacionamentos amorosos, identificação grupal, bem-estar subjetivo, diferenças psicológicas entre sexos sob a perspectiva evolucionista.

E-mail: jeannatividade@gmail.com

Fívia de Araújo Lopes é graduada em Psicologia pela UFRN (1995), com mestrado (1998) e doutorado (2002) em Psicobiologia pela UFRN. Realizou atividades de pós-doutoramento no LEEC, Universidade Paris-13 (Villetaneuse-França), é professora associada IV da UFRN no Departamento de Fisiologia, é coordenadora do PPg em Psicobiologia, e coordena também o LECH (Laboratório de Evolução do Comportamento Humano - UFRN).

E-mail: fivialopes@gmail.com

Contato com os autores:

Fívia de Araújo Lopes

UFRN - Campus Universitário

Centro de Biociências - Departamento de Fisiologia

Caixa Postal 1511 - Lagoa Nova

CEP: 59078-970

Natal-RN, Brasil 


\section{Anexo 1}

Escala Amor do Marriage and Relationships Questionnaire (MARQ) - Brasil

Por favor, leia as perguntas abaixo e responda de acordo de acordo com a escala de intensidade ao lado. Observe que 1 significa Nem um pouco e 5 significa Muito. Considere seu relacionamento atual para responder.

\begin{tabular}{lccccc}
\hline & 1 & 2 & 3 & 4 & 5 \\
\hline & Nem um pouco & & & & Muito \\
Você gosta da companhia de sua(seu) parceira(o)? & 1 & 2 & 3 & 4 & 5 \\
Você é feliz com seu relacionamento? & 1 & 2 & 3 & 4 & 5 \\
Você acha sua(seu) parceira(o) atraente? & 1 & 2 & 3 & 4 & 5 \\
Vocês gostam de fazer coisas juntos? & 1 & 2 & 3 & 4 & 5 \\
Você gosta de ficar abraçado(a) com sua(seu) parceira(o)? & 1 & 2 & 3 & 4 & 5 \\
Você respeita sua(seu) parceira(o)? & 1 & 2 & 3 & 4 & 5 \\
Você se orgulha de sua(seu) parceira(o)? & 1 & 2 & 3 & 4 & 5 \\
Seu relacionamento tem um lado romântico? & 1 & 2 & 3 & 4 & 5 \\
Quanto você ama sua(seu) parceira(o) & 1 & 2 & 3 & 4 & 5 \\
\hline
\end{tabular}

Cálculo dos níveis de vínculo emocional: calcular média aritmética dos nove itens. 
